\title{
Multi-Agent Sliding Mode Control for State of Charge Balancing Between Battery Energy Storage Systems Distributed in a DC Microgrid
}

Morstyn, Thomas; Savkin, Andrey V.; Hredzak, Branislav; Agelidis, Vassilios G.

Published in:

IEEE Transactions on Smart Grid

Link to article, DOI:

10.1109/TSG.2017.2668767

Publication date:

2018

Document Version

Peer reviewed version

Link back to DTU Orbit

Citation (APA):

Morstyn, T., Savkin, A. V., Hredzak, B., \& Agelidis, V. G. (2018). Multi-Agent Sliding Mode Control for State of Charge Balancing Between Battery Energy Storage Systems Distributed in a DC Microgrid. IEEE Transactions on Smart Grid, 9(5), 4735-4743. https://doi.org/10.1109/TSG.2017.2668767

\section{General rights}

Copyright and moral rights for the publications made accessible in the public portal are retained by the authors and/or other copyright owners and it is a condition of accessing publications that users recognise and abide by the legal requirements associated with these rights.

- Users may download and print one copy of any publication from the public portal for the purpose of private study or research.

- You may not further distribute the material or use it for any profit-making activity or commercial gain

- You may freely distribute the URL identifying the publication in the public portal 


\title{
Multi-Agent Sliding Mode Control for State of Charge Balancing Between Battery Energy Storage Systems Distributed in a DC Microgrid
}

\author{
Thomas Morstyn, Member, IEEE, Andrey V. Savkin, Senior Member, IEEE, \\ Branislav Hredzak, Senior Member, IEEE and Vassilios G. Agelidis, Fellow, IEEE
}

\begin{abstract}
This paper proposes the novel use of multi-agent sliding mode control for state of charge balancing between distributed DC microgrid battery energy storage systems. Unlike existing control strategies based on linear multi-agent consensus protocols, the proposed nonlinear state of charge balancing strategy (i) ensures the battery energy storage systems are either all charging or all discharging, thus eliminating circulating currents, increasing efficiency and reducing battery lifetime degradation, (ii) achieves faster state of charge balancing, (iii) avoids overloading the battery energy storage systems during periods of high load and (iv) provides plug and play capability. The proposed control strategy can be readily integrated with existing multi-agent controllers for secondary voltage regulation and accurate current sharing. The performance of the proposed control strategy was verified with an RTDS Technologies realtime digital simulator, using switching converter models and nonlinear lead-acid battery models.
\end{abstract}

Index Terms-Battery energy storage systems, DC microgrid, distributed energy storage, distributed sliding mode control, hybrid systems, multi-agent control, secondary control, state of charge balancing.

\section{INTRODUCTION}

$\mathbf{T}$ HE rapid adoption of distributed renewable sources is being driven by the potential for increased efficiency, increased reliability and reduced pollution compared to the traditional model of centralised power generation [1]. However, the intermittent nature of these sources introduces challenges for network power quality and stability. At the same time, technological developments and increased scales of production have driven down the price of battery energy storage technologies, making them viable for power network applications [2].

Microgrids have been proposed for organising future power networks made up of distributed renewable sources and energy storage systems [3]. A microgrid is a collocated set of generation sources, energy storage systems and loads that are able to operate autonomously [4]. In particular, DC microgrids have the advantage of requiring fewer power conversion stages when integrating modern DC-based sources (e.g. Photovolatic

T. Morstyn is the with the Department of Engineering Science at the University of Oxford, Oxford OX1 3PJ, United Kingdom. (email: thomas.morstyn@eng.ox.ac.uk)

A. V. Savkin and B. Hredzak are with the School of Electrical Engineering and Telecommunications at The University of New South Wales (UNSW Australia), Sydney, NSW 2052 Australia. (email: a.savkin@unsw.edu.au, b.hredzak@unsw.edu.au).

V. G. Agelidis is with the Department of Electrical Engineering at the Technical University of Denmark (DTU), 4000 Roskilde, Denmark. (email: vasagel@elektro.dtu.dk).
(PV), variable speed wind, batteries), increasing efficiency [5]. Also, DC microgrids have been shown to provide reliability an order of magnitude higher than AC microgrids [6].

To achieve autonomous operation, a primary load sharing control strategy is required between microgrid sources. Decentralised V-I droop control is the standard primary control strategy used in DC microgrids [7]. However, V-I droop control has several limitations when used to coordinate load sharing between battery energy storage systems (BESSs). Voltage drops across the microgrid lines will cause each battery to operate at a different charging/discharging current. This will cause the state of charge (SoC) levels of the BESSs to diverge. This is undesirable, since BESSs that have run out of charge cannot contribute their current capacities to the microgrid. Also, batteries suffer significant lifetime deterioration when overcharged or undercharged [8].

SoC balancing has been proposed as a means of fully utilising the combined power and energy capacities of distributed microgrid BESSs [9]. Under a SoC balancing control strategy, the BESSs share the microgrid load, while using their excess current capacities to move towards a balanced SoC. Once a balanced SoC is achieved, the combined current capacity of the BESSs is available to balance the microgrid load. None of the BESSs prematurely run out of energy. Also, the maximum depth of discharge reached by the BESSs is reduced, improving lifetime and efficiency.

A centralised control strategy can be used to provide $\mathrm{SoC}$ balancing between microgrid BESSs [10], [11]. However, a central controller introduces a single point of failure, and the communications and processing infrastructure for centralised SoC estimation and control may be impractical for microgrids with many small distributed BESSs [12]. Also, data centralisation introduces privacy and security concerns [13].

Multi-agent control provides a scalable means of organising cooperation between microgrid BESSs, without requiring a central controller. Under a multi-agent control strategy, autonomous agents use local information and neighbour-toneighbour communication to cooperatively achieve common objectives [14].

Multi-agent control strategies have been proposed for a range of microgrid applications, including secondary control [15]-[17] and economic dispatch [18]-[20]. In [21], a control strategy is presented to coordinate the operating modes of renewable sources and BESSs in an islanded DC microgrid. When the batteries approach full charge, renewable sources use 


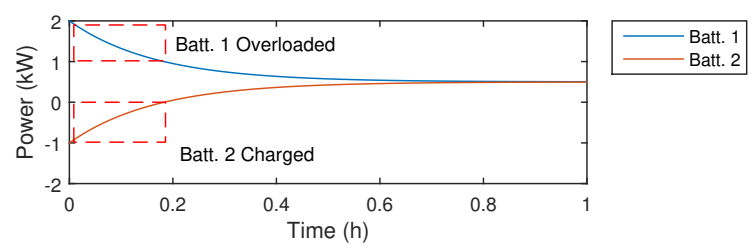

(a) Battery output powers.

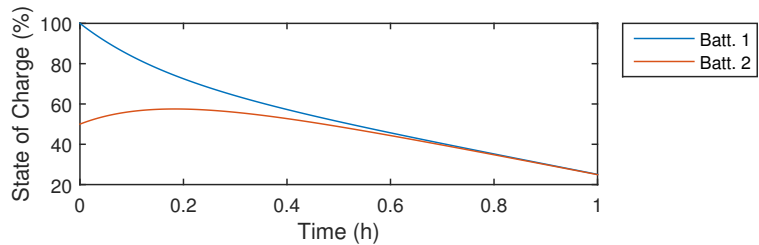

(b) Battery SoC levels.

Fig. 1. Microgrid with two $2 \mathrm{kWh}, 1 \mathrm{~kW}$ rated BESSs operating under a linear SoC balancing strategy with high gain. Initially BESS 1 is overloaded. Also, BESS 2 is charged, resulting in circulating currents.

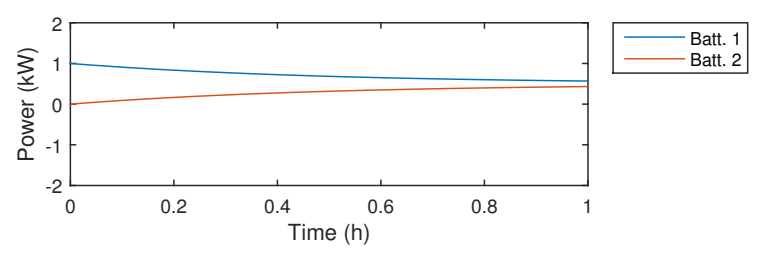

(a) Battery output powers.

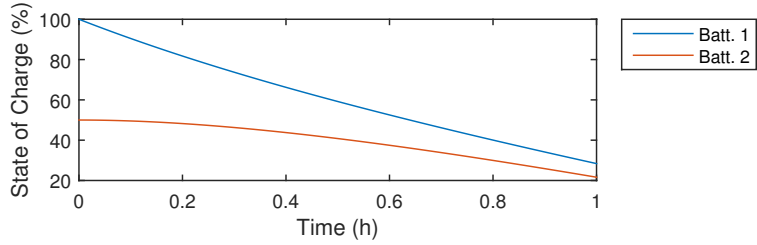

(b) Battery SoC levels.

Fig. 2. Microgrid with two $2 \mathrm{kWh}, 1 \mathrm{~kW}$ rated BESSs operating under a linear SoC balancing strategy, with the maximum gain possible without overloading battery 1 . Since only a fraction of the battery power capacity is used for most of the operating time, SoC balancing is very slow.

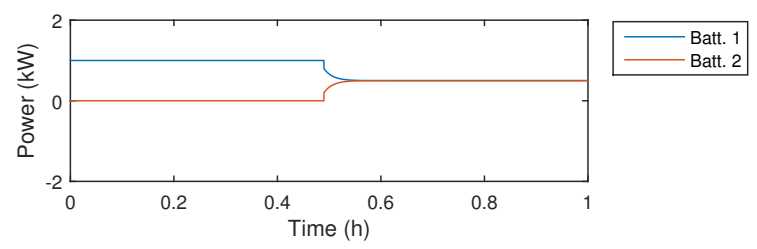

(a) Battery output powers.

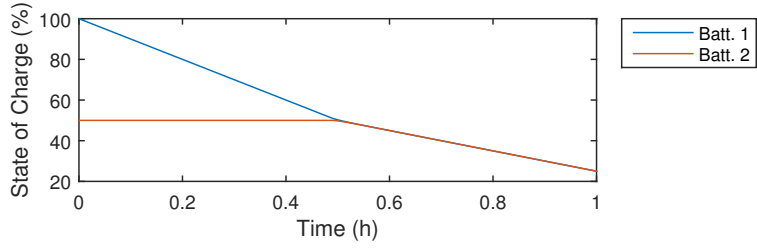

(b) Battery SoC levels.

Fig. 3. Microgrid with two $2 \mathrm{kWh}, 1 \mathrm{~kW}$ rated BESSs operating under the proposed desirable nonlinear SoC balancing strategy.

curtailment to regulate the microgrid voltage. Communication between the BESSs is provided by a power line signalling method, which entails all-to-all (rather than neighbour-toneighbour) communication. A review of DC microgrid control strategies, including distributed multi-agent approaches, is presented in [22]. The rigorous mathematical analysis of multi-agent control strategies is identified as a key research challenge.

Multi-agent SoC balancing control strategies for distributed microgrid BESSs are presented in [9], [23]-[28]. For DC microgrids, multi-agent SoC balancing has been combined with multi-agent secondary voltage control [23] and extended to coordinate heterogeneous energy storage technologies [24].

Existing multi-agent SoC balancing strategies [9], [23]-[28] use linear consensus protocols. This introduces an undesirable trade-off between the rate of SoC balancing and the utilisation of the BESS current capacities. To demonstrate the limitations of a linear consensus strategy, consider the idealised example of a microgrid with two $2 \mathrm{kWh}, 1 \mathrm{~kW}$ rated BESSs and a $1 \mathrm{~kW}$ load. Fig. 1(a) and Fig. 1(b) show the battery output powers and SoC levels, under a linear consensus strategy with high gain. Initially, BESS 1 is overloaded and BESS 2 operates in charging mode. The circulating currents between the BESSs will result in lower efficiency, and lifetime degradation due to unnecessary cycling of the batteries. Fig. 2(a) and Fig. 2(b) show the battery output powers and SoC levels when the gain is reduced to ensure the BESSs are not overloaded. In this case, the rate of SoC balancing is very slow since the full power capacities of the batteries are not utilised.

The desirable operation shown in Fig. 3(a) and Fig. 3(b) requires a nonlinear control strategy. BESS 1 operates at its maximum output power to supply the full microgrid load until a balanced SoC is reached. During this time, BESS 2 operates at zero output power, so there are no circulating currents. This paper proposes a nonlinear control strategy that achieves this desired operation.

This paper proposes the novel use of multi-agent sliding mode control for SoC balancing between distributed DC microgrid BESSs. The proposed multi-agent sliding mode control strategy provides qualitative improvements over existing SoC balancing strategies based on linear consensus protocols, namely:

(i) No Circulating Currents: The microgrid batteries are either all charging, or all discharging, depending on the net microgrid load. This prevents circulating currents and unnecessary charge/discharge cycles, improving efficiency and battery lifetime.

(ii) Fast SoC Balancing: During periods of low load, BESSs with low SoC do not participate in the droop control. This provides fast SoC balancing without introducing circulating currents.

(iii) No Overload: During periods of high load, all of the BESSs share the microgrid load within their current capacities, so that none are overloaded. The BESSs continue 


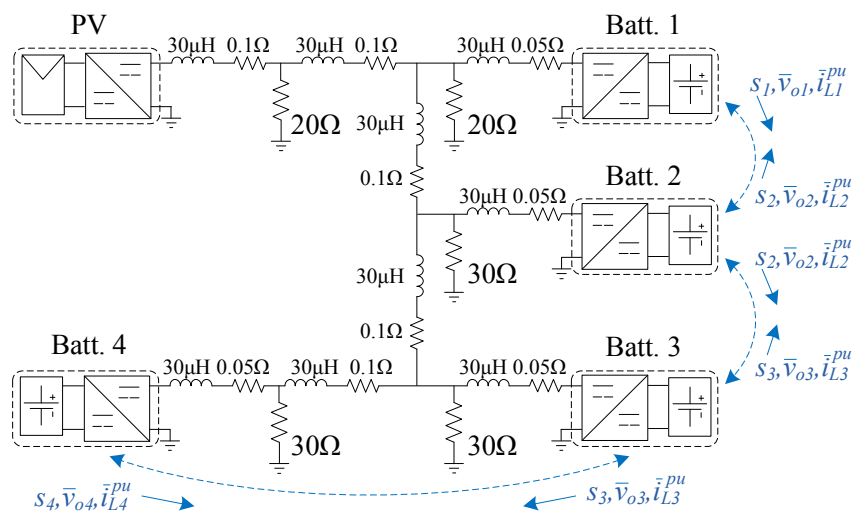

Fig. 4. Islanded 100V DC microgrid, with four lead-acid BESSs, five resistive loads and a PV generation source, connected together by RL lines. The BESSs are connected by a sparse communication network, allowing neighbour-toneighbour communication.

to move towards a balanced SoC at a reduced rate.

(iv) Plug and Play: The distributed sliding mode controllers used for SoC balancing do not need system parameter information, and do not need information on the number of BESSs. This provides plug and play capability, i.e. proper operation is maintained when BESSs connect/disconnect from the communication network and microgrid.

The SoC balancing control is integrated with existing multiagent secondary control strategies for secondary voltage regulation and accurate current sharing. Once a battery's SoC reaches a boundary layer around the average of its neighbours' SoC levels, its nonlinear sliding mode controller swaps to a linear interpolation that prevents chattering. The performance of the proposed control strategy was verified with an RTDS Technologies real-time digital simulator for a DC microgrid with PV generation and lead-acid BESSs, using switching converter models and nonlinear lead-acid battery models.

The rest of this paper is organised as follows. Section II presents the proposed multi-agent sliding mode control strategy. Section III provides real-time digital simulation results verifying the performance of the proposed control strategy. Section IV concludes the paper. In the appendix, the conditions for SoC synchronisation between BESSs operating under the proposed control strategy are rigorously analysed.

\section{PRINCIPLE OF OPERATION}

This study considers a DC microgrid with distributed BESSs $\mathcal{V}=\{1, \ldots, N\}$, each consisting of a battery connected to the microgrid through a controllable bidirectional DC-DC converter. The BESSs are connected by a sparse communication network allowing bidirectional neighbour-to-neighbour communication between them. In particular, Fig. 4 shows the islanded DC microgrid considered in this study, which has a PV generation source and four BESSs. Fig. 5 shows the topology of the individual BESSs.

On the primary control level, the standard V-I droop control is used for decentralised load sharing to maintain the microgrid power balance. A secondary control level is introduced to regulate the average BESS output voltage to the nominal

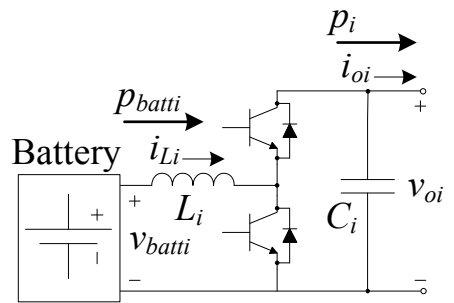

Fig. 5. BESS consisting of a battery and bidirectional DC-DC boost converter.

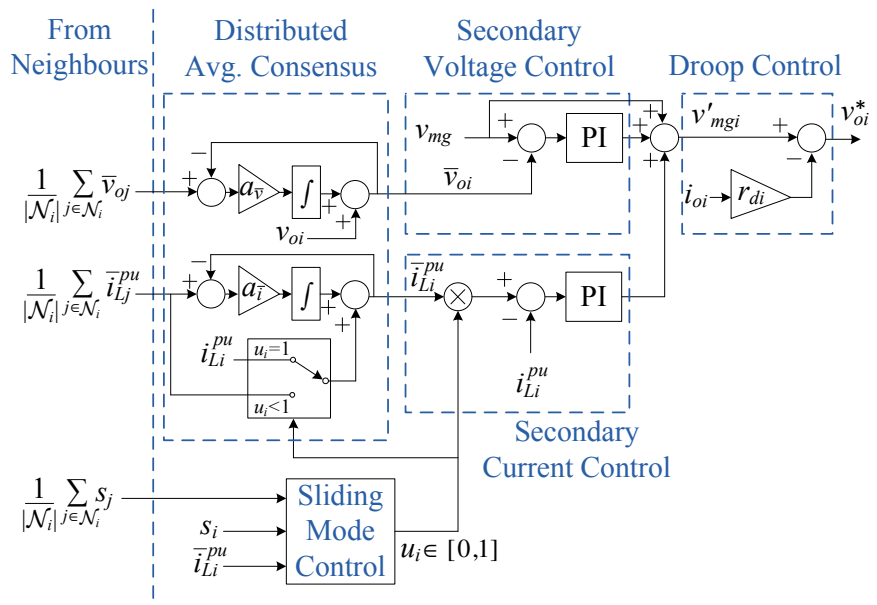

Fig. 6. Block diagram of the proposed control strategy for one of the BESSs. The proposed control strategy includes a distributed sliding mode controller for SoC balancing, added to distributed secondary voltage and current controllers. V-I droop control generates the voltage reference for the lower level voltage/current controllers.

microgrid voltage on a slower time-scale, while providing accurate current sharing between the BESSs. In particular, the multi-agent implementation of the secondary control level from [17] is used. This paper proposes a modification to the multi-agent secondary control level, with distributed sliding mode controllers introduced to provide SoC balancing between the BESSs.

Fig. 6 shows a block diagram of the proposed control strategy for one of the BESSs.

\section{A. Decentralised Primary Control}

Decentralised load sharing between the BESSs is provided by the standard V-I droop control,

$$
v_{o i}^{*}(t)=v_{m g i}^{\prime}(t)-r_{d i} i_{o i}(t) .
$$

$v_{o i}^{*}$ is the BESS output voltage reference, $i_{o i}$ is the output current and $r_{d i}$ is the droop coefficient. $v_{m g i}^{\prime}$ is a modified version of the nominal microgrid voltage $v_{m g} . v_{m g i}^{\prime}$ is set by the multi-agent secondary level control strategy to achieve secondary voltage control and SoC balancing between the BESSs.

The output voltage reference $v_{o i}^{*}$ is sent to the lower level cascaded voltage and current controllers from [29], which generate the duty cycle for pulse width modulation of the bidirectional DC-DC converter switches. 
The decentralised droop control only provides approximate current sharing between the BESSs, and will introduce steady state voltage offsets. Although voltage differences between the BESSs are inevitable due to line resistances, the voltage levels should vary around the nominal microgrid voltage.

This motivates the introduction of a DC microgrid secondary control level. On a slower time-scale than the primary control, the secondary control level strategy restores the average voltage to the nominal microgrid voltage and modifies the BESSs output currents for accurate current sharing, by adjusting the modified nominal microgrid voltage $v_{m g i}^{\prime}$. This is described in detail in Section II-B. Section II-C describes the proposed multi-agent sliding mode control strategy, which is added to the secondary control level to provide SoC balancing between the BESSs.

\section{B. Multi-Agent Secondary Control for Average Voltage Regu- lation and Accurate Current Sharing}

In this study, the proposed multi-agent sliding mode control strategy for SoC balancing between the BESSs is integrated with the multi-agent secondary control strategy from [17], for accurate current sharing and voltage restoration.

Let the communication network between the BESSs be represented by a connected undirected graph $\mathcal{G}(\mathcal{V}, \mathcal{E}) . \mathcal{E}$ is the set of graph edges, where the unordered pair $\{i, j\} \in \mathcal{E}$ if there is a communication link between BESSs $i$ and $j$. Let $\mathcal{N}_{i}$ be the neighbour set of $\operatorname{BESS} i$, where $j \in \mathcal{N}_{i}$ if $\{i, j\} \in \mathcal{E}$.

Each BESS implements the distributed dynamic average consensus protocol from [30], using local information and information from their neighbours to update a local estimate of the average output voltage $\bar{v}_{o i}$,

$$
\bar{v}_{o i}(t)=v_{o i}(t)+\frac{a_{\bar{v}}}{\left|\mathcal{N}_{i}\right|} \int_{0}^{t} \sum_{j \in \mathcal{N}_{i}}\left(\bar{v}_{o j}(\tau)-\bar{v}_{o i}(\tau)\right) d \tau .
$$

$\left|\mathcal{N}_{i}\right|$ is the number of neighbours of BESS $i$ and $a_{\bar{v}}$ is the average voltage consensus gain. Under this protocol, for step changes in the BESS output voltages, $\lim _{t \rightarrow \infty}\left(\bar{v}_{o i}-\right.$ $\left.\frac{1}{N} \sum_{j=1}^{N} v_{o j}\right)=0, \forall i \in \mathcal{V}$.

The BESSs also keep estimates of the average per-unit battery current of the BESSs that are fully participating in the droop control $\bar{i}_{L i}^{p u}$. To achieve SoC balancing, the local sliding mode controller generates a control signal $u_{i} \in[0,1]$ which sets the BESS's level of participation in the droop control (the mechanism for this is described in detail in Section II-C). Full participation is given by $u_{i}=1$. The dynamic average current consensus protocol is designed so that BESSs which are not fully participating in the droop control $\left(u_{i}<1\right)$ pass through the average of their neighbours' estimates, rather than using their own battery current as an input.

The distributed dynamic average current consensus protocol is given by,

$$
\begin{aligned}
\bar{i}_{L i}^{p u}(t) & =z_{i}(t)+\frac{a_{\bar{i}}}{\left|\mathcal{N}_{i}\right|} \int_{0}^{t} \sum_{j \in \mathcal{N}_{i}}\left(\bar{i}_{L j}^{p u}(\tau)-\bar{i}_{L i}^{p u}(\tau)\right) d \tau \\
z_{i}(t) & = \begin{cases}i_{L i}^{p u}(t), & u_{i}(t)=1 \\
\frac{1}{\left|\mathcal{N}_{i}\right|} \sum_{j \in \mathcal{N}_{i}} \bar{i}_{L j}^{p u}(t), & u_{i}(t)<1 .\end{cases}
\end{aligned}
$$

$a_{\bar{i}}$ is the average current consensus gain. The per-unit battery current $i_{L i}^{p u}=i_{L i} / C_{b a t t i}$ (i.e. the battery current divided by the charge capacity) is used, to account for BESSs with different charge capacities. Let the set of BESSs that are fully participating in the droop control be given by $\mathcal{P}=$ $\left\{i \in \mathcal{V} \mid u_{i}=1\right\}$. For step changes in the battery currents, $\lim _{t \rightarrow \infty}\left(\bar{i}_{L i}^{p u}-\frac{1}{|\mathcal{P}|} \sum_{j \in \mathcal{P}} i_{L j}^{p u}\right)=0, \forall i \in \mathcal{V}$.

The nominal microgrid voltage used by the droop control $v_{m g i}^{\prime}$ is modified by PI controllers for secondary voltage and current control.

$v_{m g i}^{\prime}(t)=v_{m g}+k_{p}^{\bar{v}} e_{i}^{\bar{v}}(t)+k_{p}^{\bar{i}} e_{i}^{\bar{i}}(t)+\int_{0}^{t} k_{i}^{\bar{v}} e_{i}^{\bar{v}}(\tau)+k_{i}^{\bar{i}} e_{i}^{\bar{i}}(\tau) d \tau$,

$e_{i}^{\bar{v}}(t)=v_{m g}-\bar{v}_{o i}(t), e_{i}^{\bar{i}}(t)=u_{i}(t) \bar{i}_{L i}^{p u}(t)-i_{L i}^{p u}(t)$.

$k_{p}^{\bar{v}}$ and $k_{i}^{\bar{v}}$ are the secondary voltage control proportional and integral gains. $k_{p}^{\bar{i}}$ and $k_{i}^{\bar{i}}$ are the secondary current control proportional and integral gains.

Under the secondary control strategy (4), $v_{m g i}^{\prime}$ is increased if the local estimate of the average BESS output voltage $\bar{v}_{o i}$ is below the nominal microgrid voltage $v_{m g}$, and decreased if $\bar{v}_{o i}$ is above $v_{m g}$. This regulates the average of the BESS output voltages $\frac{1}{N} \sum_{i=1}^{N} v_{o i}$ to the nominal microgrid voltage $v_{m g}$ [17]. $v_{m g i}^{\prime}$ is also adjusted for secondary current control. When a BESS is fully participating in the droop control $\left(u_{i}=1\right)$, its per-unit battery current $i_{L i}^{p u}$ is regulated to the local estimate of the average per-unit current of the participating batteries $\bar{i}_{L i}^{p u}$. When a BESS is not participating in the droop control $\left(u_{i}=0\right)$, its battery current is regulated to zero.

\section{Multi-Agent Sliding Mode Control for State of Charge Balancing}

The BESS's local sliding mode controller generates a control signal $u_{i}$ that determines its level of participation in the droop control to achieve SoC balancing, based on the local SoC $s_{i}$, and the average SoC of its neighbours. The average SoC of the neighbours of BESS $i$ at time $t$ is defined as

$$
\mathcal{A}_{i}(t):=\frac{1}{\left|\mathcal{N}_{i}\right|} \sum_{j \in \mathcal{N}_{i}} s_{j}(t)
$$

First, the following (idealised) sliding mode control function is proposed,

$$
u_{i}(t)= \begin{cases}1, & s_{i}(t) \geq \mathcal{A}_{i}(t) \text { and } \bar{i}_{L i}^{p u}(t)>0 \\ 1, & s_{i}(t) \leq \mathcal{A}_{i}(t) \text { and } \bar{i}_{L i}^{p u}(t)<0 \\ 0, & \text { otherwise }\end{cases}
$$

The BESS will fully participate in the droop control $\left(u_{i}=1\right)$ if, (a) the microgrid has a net load $\left(\bar{i}_{L i}^{p u}>0\right)$ and the local SoC is greater than, or equal to, the average of the neighbours' SoC levels, or (b) the microgrid has net generation $\left(\bar{i}_{L i}^{p u}<0\right)$ and the local SoC is less than, or equal to, the average of the neighbours' SoC levels.

The key idea behind the design of the sliding mode control function is that the BESSs always move closer to the average SoC of their neighbours, or keep the same SoC. The appendix provides a rigorous analysis of the conditions under which the proposed multi-agent sliding mode control strategy provides SoC synchronisation. 


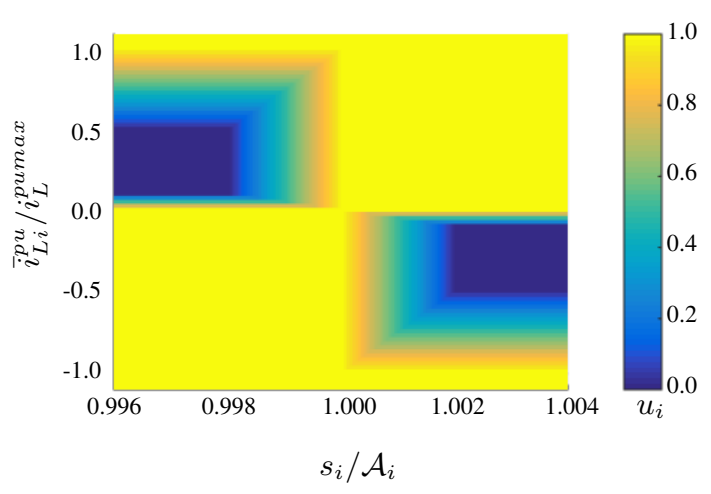

Fig. 7. Sliding mode control function $u_{i}\left(s_{i}, \mathcal{A}_{i}, \bar{i}_{L i}^{p u}\right)$.

A direct implementation of the sliding mode control function (6) would have two problems. First, only a subset of the BESSs participate in the droop control. During high load conditions, these BESSs could be overloaded. Second, as a BESS's SoC approaches the average SoC of its neighbours, the sliding mode control signal switches rapidly, resulting in chattering and excessive battery current variations.

Fig. 7 shows a modified implementation of the sliding mode control function (6). The sliding mode control prioritises overload prevention over SoC balancing. This is done by ensuring that all of the batteries participate in the droop control if the average per-unit current of the participating BESSs exceeds a common maximum per-unit charge/discharge rate $i_{L}^{\text {pumax }}=i_{L i}^{\max } / C_{\text {batti }}, \forall i \in \mathcal{V}$, i.e. $u_{i}=1$ if $\left|\bar{i}_{L i}^{p u}\right|>i_{L}^{\text {pumax }}$.

To prevent chattering, boundary layers are introduced to smooth out the discontinuities in the distributed sliding mode controllers. In the boundary layers, the sliding mode control signal is given by a linear interpolation between the values it would otherwise take [31]. Boundary layers are included for the transitions from $s_{i}<\mathcal{A}_{i}$ to $s_{i}>\mathcal{A}_{i}, \bar{i}_{L i}^{p u}<0$ to $\bar{i}_{L i}^{p u}>0$ and $\left|\bar{i}_{L i}^{p u}\right|<i_{L}^{\text {pumax }}$ to $\left|\bar{i}_{L i}^{p u}\right|>i_{L}^{\text {pumax }}$. The boundary layers prevent rapid battery current variations, but at the cost of SoC synchronisation accuracy.

The distributed dynamic average consensus protocols (2), (3) and multi-agent sliding mode control (6), only require that a sparse undirected communication network is maintained between the microgrid BESSs. As shown in Fig. 4, the BESSs use neighbour-to-neighbour communication to share their SoC, their estimates of the average output voltage, and their estimates of the average per-unit battery current of the BESSs fully participating in the droop control. In particular, the BESSs do not require information on the number of BESSs in the microgrid, or the microgrid topology. When a BESS disconnects/connects to the microgrid and communication network, only its direct neighbours need to update their local control strategies. This provides plug-and-play capability.

\section{RESULTS}

To verify the performance of the proposed control strategy, it was implemented for the $100 \mathrm{~V}$ islanded DC microgrid shown in Fig. 4, using an RTDS Technologies real-time digital simulator, with switching converter models and nonlinear battery models from [32]. Using switching converter models
TABLE I

CASE Study PARAMETERs

\begin{tabular}{llllll}
\hline$v_{m g}$ & $100 \mathrm{~V}$ & $r_{d}$ & 1 & $v_{\text {batt } 1,2,3,4}^{[\text {full }]}$ & $52 \mathrm{~V}$ \\
$E_{\text {batt } 1,2}$ & $1.06 \mathrm{kWh}$ & $C_{\text {batt } 1,2}$ & $20.4 \mathrm{Ah}$ & $i_{L 1,2}^{\max }$ & $20 \mathrm{~A}$ \\
$E_{\text {batt } 3,4}$ & $0.56 \mathrm{kWh}$ & $C_{\text {batt } 3,4}$ & $10.8 \mathrm{Ah}$ & $i_{L 3,4}^{\max }$ & $10.6 \mathrm{~A}$ \\
$a_{\bar{v}}$ & 10 & $k_{\bar{p}}^{\bar{v}}$ & 0.1 & $k_{i}^{\bar{v}}$ & 0.5 \\
$a_{\bar{i}}$ & $2 \times 10^{2}$ & $k_{p}^{\bar{i}}$ & 0.4 & $k_{i}^{\bar{i}}$ & 20 \\
$f_{d c d c}$ & $2 \mathrm{kHz}$ & $L$ & $10 \mathrm{mH}$ & $C$ & $3.3 \mathrm{mF}$ \\
\hline
\end{tabular}

and accurate battery models, capturing the fast and slow timescale voltage and SoC dynamics, allows the correct interaction between the microgrid control levels to be verified.

The case study parameters are provided in Table I. The DC microgrid has four lead-acid BESSs, five resistive loads $(2 \mathrm{~kW}$ total at $100 \mathrm{~V})$ and a PV generation source, connected together by RL lines. BESS 1 and BESS 2 have $1.06 \mathrm{kWh}$, $52 \mathrm{~V}$ lead-acid batteries, with charge/discharge limits of $\pm 20 \mathrm{~A}$. BESS 3 and BESS 4 have $0.56 \mathrm{kWh}, 52 \mathrm{~V}$ lead-acid batteries, with charge/discharge limits of $\pm 10.6 \mathrm{~A}$. Fig. 4 also shows the sparse communication network allowing neighbour-toneighbour communication between the BESSs. The communication network has $10 \mathrm{~ms}$ delays.

Two case studies are presented. Case Study A uses a step generation profile to clearly demonstrate the features of the proposed control strategy. Case Study B uses a realistic PV generation profile based on one minute resolution temperature and irradiance data.

\section{A. Case Study A. Step Generation Profile}

First, the step generation profile shown in Fig. 8(a) is used, to clearly demonstrate the features of the proposed control strategy, namely: (i) No circulating currents, (ii) fast SoC balancing, (iii) no overload and (iv) plug and play capability.

As shown in Fig. 8(b), the microgrid BESSs begin with SoC levels between $40 \%$ and $70 \%$. The output currents of the batteries are shown in Fig. 8(c).

Initially, the PV source operates at zero output power, and thus the full microgrid load must be provided by the BESSs. BESS 1 has high SoC relative to its neighbour, BESS 2 $\left(s_{1}(0)=70 \%\right.$ and $\left.s_{2}(0)=60 \%\right)$, and the microgrid has a net load, so it fully participates in the droop control $\left(u_{1}(0)=1\right)$. BESS 4 has low SoC relative to its neighbour, BESS 3 $\left(s_{4}(0)=40 \%\right.$ and $\left.s_{3}(0)=50 \%\right)$. However, the discharging currents required to feed the load mean that all of the BESSs must contribute to the droop control to prevent BESS 1 from being overloaded. BESS 4 operates in the boundary layer between $\bar{i}_{L 4}^{p u}<i_{L 4}^{\max } / C_{\text {batt } 4}^{\max }$ and $\bar{i}_{L 4}^{p u}>i_{L 4}^{\max } / C_{\text {batt } 4}^{\max }$. BESSs 2 and 3 have SoC levels close to the average of their neighbours, $\left(s_{2}(0)=\frac{s_{1}(0)+s_{3}(0)}{2}=60 \%\right.$ and $\left.s_{3}(0)=\frac{s_{2}(0)+s_{4}(0)}{2}=50 \%\right)$. Their sliding mode controllers enter the boundary layer between $s_{i}<\mathcal{A}_{i}$ and $s_{i}>\mathcal{A}_{i}$, and these BESSs operate with output currents between BESS 1 and BESS 4 .

When the net microgrid load is high, the rate of SoC balancing is low, since all of the BESSs must at least partially participate in the droop control to prevent an overload from 


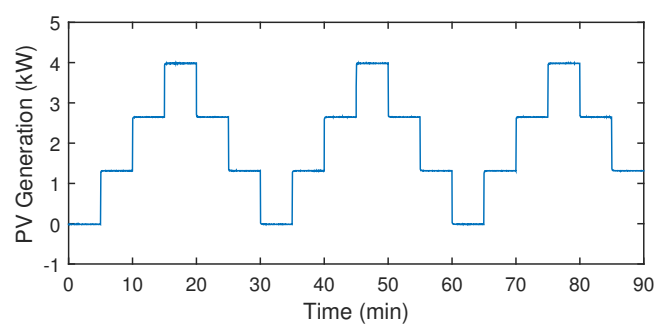

(a) PV source output power.

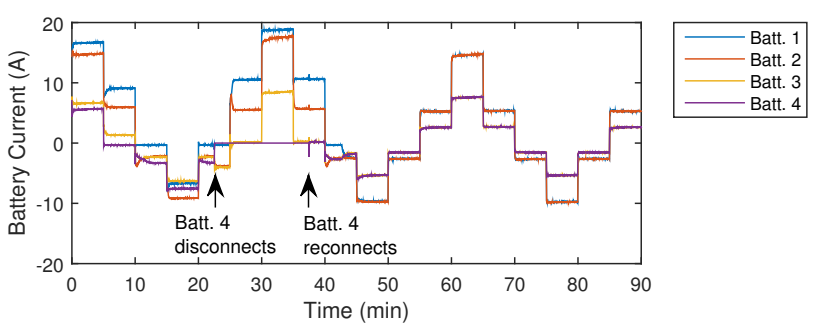

(c) Battery output currents.

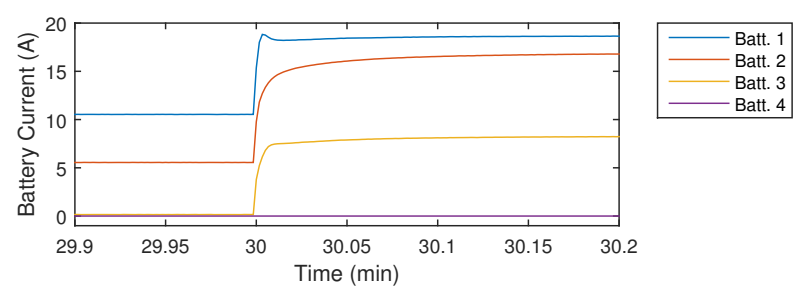

(e) Transient battery output currents (29.9 minutes to 30.2 minutes).

Fig. 8. Case Study A. Step generation profile.

occurring. When the output power of the PV source increases, the batteries can operate with a larger range of discharging currents. The desired behaviour from Fig. 3, with the BESSs moving to a balanced SoC, and the lowest SoC BESS operating at zero output power, is demonstrated in Case Study A between 5 minutes and 10 minutes.

The range of charging/discharging currents, and thus the rate of SoC balancing, is limited by the requirement that all of the BESSs are discharged when the microgrid has a net load, and charged when the microgrid has net generation. This ensures there are no circulating currents, increasing efficiency and preventing unnecessary charge/discharge cycles. As demonstrated by the examples in Fig. 1 and Fig. 2, if instead a multi-agent SoC balancing strategy based on linear consensus protocols was used, it would present an undesirable choice between overloads during the periods of high load (e.g. 0 minutes to 5 minutes) and circulating currents during periods of low load (e.g. 5 minutes to 10 minutes), or slow SoC balancing, depending on the gain selected.

An advantage of the multi-agent control structure is the plug and play nature of the system. To demonstrate this capability, BESS 4 is commanded to disconnect from the microgrid and the communication network at 22.5 minutes, and to reconnect at 37.5 minutes. As shown in Fig. 8(c), BESS 4 goes from charging to zero output current. Fig. 8(d) shows that this causes an increase in the microgrid voltages. BESS 2 and 3 respond by increasing their charging rate to restore the

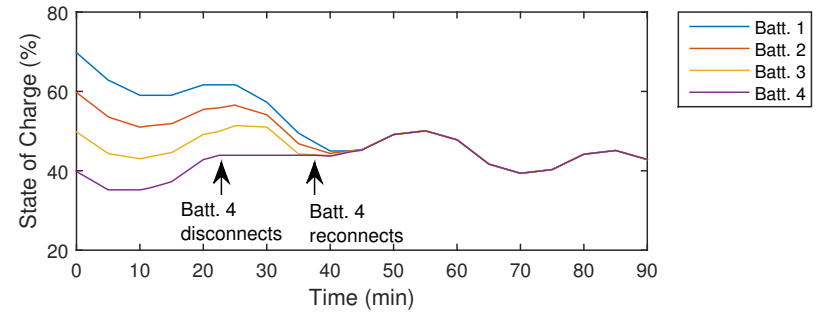

(b) Battery SoC levels.

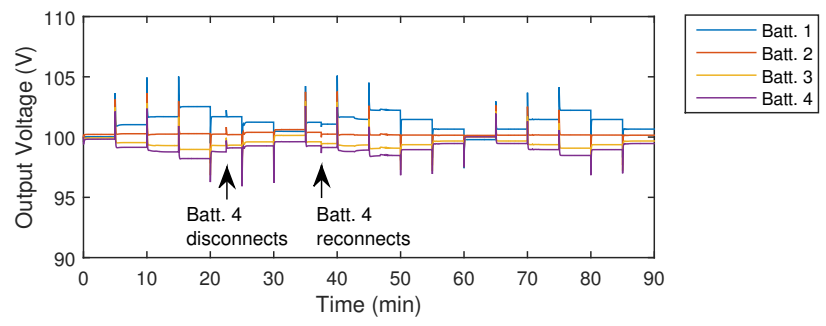

(d) BESS output voltages.
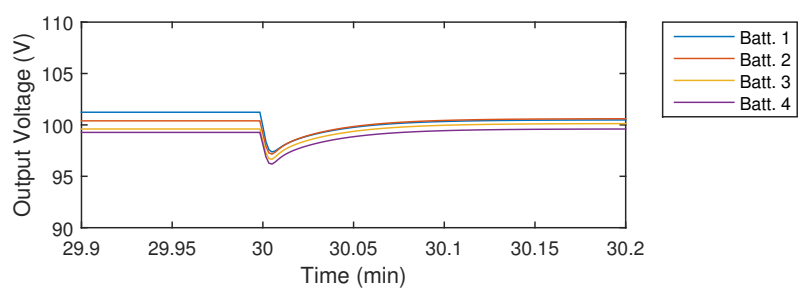

(f) Transient BESS output voltages (29.9 minutes to 30.2 minutes).

microgrid power balance, while BESS 1 remains at zero output current, since it has high SoC. Fig. 8(b) shows that while BESS 4 is disconnected, its SoC remains unchanged. The remaining BESSs pursue SoC between themselves, since BESS 4 is not included in the communication network.

The BESSs reach an approximately balanced SoC after 50 minutes. Exact SoC balancing is not achieved, due to the linear boundary layers of the sliding mode controllers. However, after this time the difference between the highest and lowest battery SoC is less than $0.04 \%$.

As shown in Fig. 8(d), transient voltage changes occur due to the primary V-I droop control when the PV source changes its output power. On a slower time-scale, the multiagent secondary voltage control regulates the average of the BESS output voltages to the $100 \mathrm{~V}$ reference. Fig. 8(e) and Fig. 8(f) show the transient behaviour of the battery output currents and BESS output voltages between 29.9 minutes and 30.2 minutes.

\section{B. Case Study B. Realistic PV Generation Profile}

To verify the performance of the proposed control strategy for a realistic senario, a PV generation profile was used, based on one minute resolution temperature and irradiance data from the NREL Baseline Measurement System in Colorado, for 11 am to $12: 30 \mathrm{pm}$ on July $2^{\text {nd }} 2015$, shown in Fig. 9(a).

Fig. 9(b) shows the BESS SoC levels, Fig. 9(c) shows the battery output currents and Fig. 9(d) shows the BESS output 


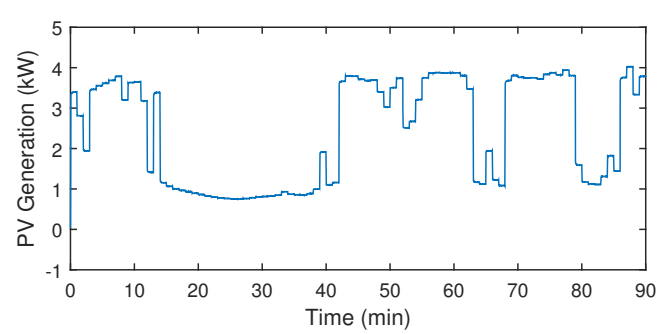

(a) PV source output power.

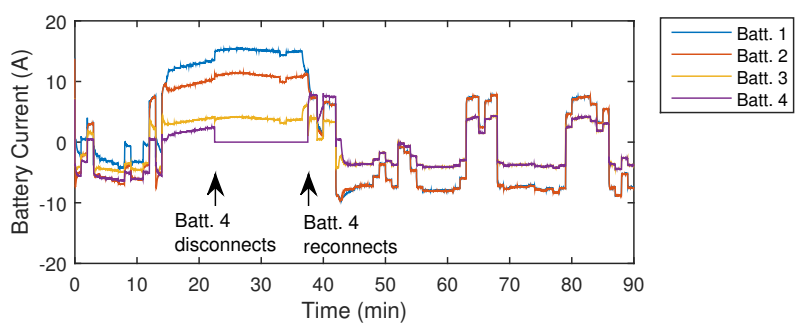

(c) Battery output currents.

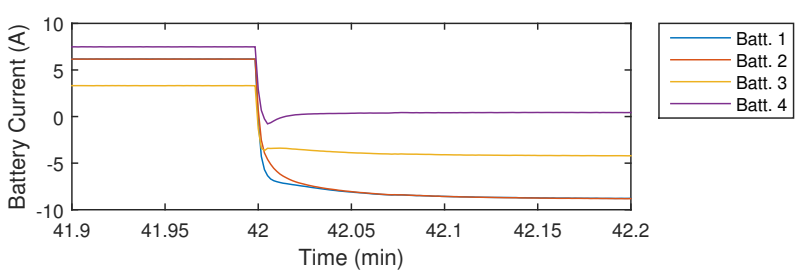

(e) Transient battery output currents (41.9 minutes to 42.2 minutes).

Fig. 9. Case Study B. Realistic PV generation profile.

voltages. Fig. 9(e) and Fig. 9(f) show the transient behaviour of the battery output currents and BESS output voltages between 41.9 minutes and 42.2 minutes. As in Case Study A, (i) no circulating currents, (ii) fast SoC balancing, (iii) no overload and (iv) plug and play capability are all demonstrated.

\section{CONCLUSION}

This paper has presented a multi-agent sliding mode control strategy for SoC balancing between distributed DC microgrid BESSs. The proposed control strategy provides significantly improved operation compared to existing SoC balancing strategies based on linear consensus protocols. By utilising the full excess capacity of the BESSs, fast SoC balancing is achieved without circulating currents. Eliminating circulating currents increases efficiency and reduces battery lifetime degradation. Real-time digital simulation results have been presented demonstrating the performance of the proposed control strategy.

\section{APPENDIX}

This appendix provides a rigorous analysis of the conditions under which the proposed multi-agent sliding mode control strategy provides SoC synchronisation between the distributed DC microgrid BESSs.

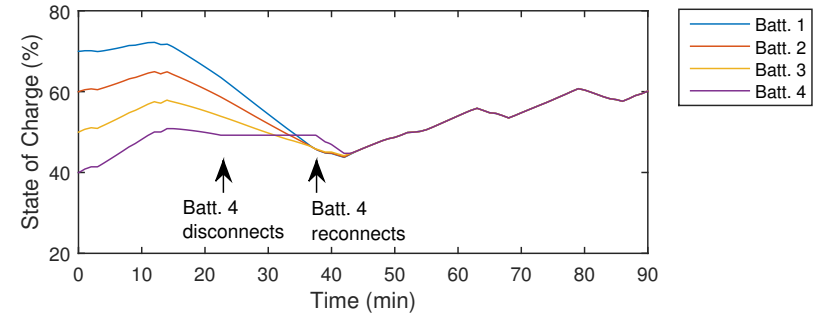

(b) Battery SoC levels.

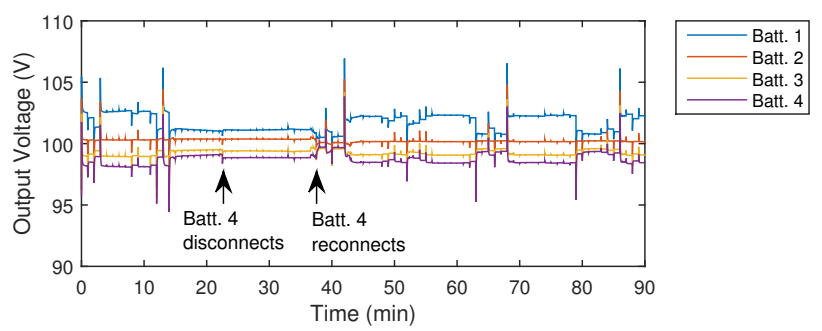

(d) BESS output voltages.

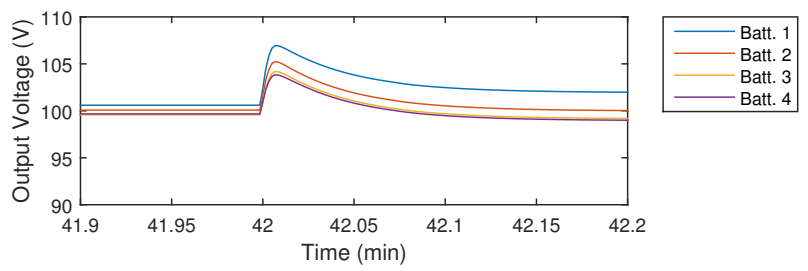

(f) Transient BESS output voltages (41.9 minutes to 42.2 minutes).

The dynamics of each BESS $i \in \mathcal{V}$ are described by the following system of nonlinear equations:

$$
\begin{array}{r}
\dot{x}_{i}(t)=f_{i}\left(x_{i}, u_{i}\right), \\
s_{i}(t)=r_{i}^{T} x_{i}(t),
\end{array}
$$

where $x_{i}(t) \in \mathbf{R}^{m_{i}}$ is the state of BESS $i, u_{i}(t) \in \mathbf{R}$ is the charging/discharging control signal and $s_{i}(t) \in \mathbf{R}$ is the SoC of BESS $i$. In this case, the variation of the SoC is described by the equation

$$
\dot{s}_{i}(t)=r_{i}^{T} f_{i}\left(x_{i}, u_{i}\right)
$$

It is assumed that for all $i \in \mathcal{V}$ and all $x_{i} \in \mathbf{R}^{m_{i}}$,

$$
r_{i}^{T} f_{i}\left(x_{i}, 0\right)=0 .
$$

Also, it is assumed that there exist functions $U_{1}\left(i_{L}^{p u}, x\right), U_{2}\left(i_{L}^{p u}, x\right), \ldots, U_{N}\left(i_{L}^{p u}, x\right) \quad$ defined for all $i_{L}^{p u} \in\left[-i_{L}^{\text {pumax }}, i_{L}^{\text {pumax }}\right]$ and $x_{i} \in \mathbf{R}^{m_{i}}$ such that,

$$
-i_{L}^{p u}=r_{i}^{T} f_{i}\left(x_{i}, U_{i}\left(i_{L}^{p u}, x\right)\right) .
$$

Let $p_{i}(t)=g_{i}\left(x_{i}, u_{i}\right)$ be the output power of BESS $i$,

It is assumed that the batteries have charging and discharging efficiencies between $0 \%$ and $100 \%$. Let $\epsilon_{0}>0$ be some given constant. Then,

$$
\begin{gathered}
\dot{s}_{i}(t) \leq-\frac{p_{i}(t)}{E_{\text {batti }}} \leq\left(1+\epsilon_{0}\right) \dot{s}_{i}(t) \quad \text { if } \quad \dot{s}_{i}(t)>0, \\
\dot{s}_{i}(t) \leq-\frac{p_{i}(t)}{E_{\text {batti }}} \leq\left(1-\epsilon_{0}\right) \dot{s}_{i}(t) \quad \text { if } \quad \dot{s}_{i}(t)<0 .
\end{gathered}
$$


$E_{\text {batti }}$ is the battery energy capacity. The difference between $\dot{s}_{i}(t)$ and $-\frac{p_{i}(t)}{E_{\text {batti }}}$ is the per-unit charge loss of the charge/discharge process.

Let $P_{n e t}^{p u}(t):=\sum_{i=1}^{N} \frac{p_{i}(t)}{E_{\text {batti }}}$ be the net microgrid load/generation, normalised by the battery energy capacities. Also, let $S_{0}:=\sum_{i=1}^{N} s_{i}(0)$ and,

$$
\hat{P}_{n e t}^{p u}(t):= \begin{cases}\frac{1}{1+\epsilon_{0}} P_{n e t}^{p u}(t), & P_{n e t}^{p u}(t) \leq 0, \\ \frac{1}{1-\epsilon_{0}} P_{n e t}^{p u}(t), & P_{n e t}^{p u}(t)>0 .\end{cases}
$$

The following constraints on the SoC of the BESSs must be satisfied, to prevent overcharging/undercharging:

$$
s^{\min } \leq s_{i}(t) \leq s^{\max }, \forall i \in \mathcal{V},
$$

with given constants $s^{\max }>s^{\min }>0$.

To satisfy this requirement, the following assumptions are required: (a) The BESSs must begin within their allowed SoC ranges,

$$
s^{\min }<s_{i}(0)<s^{\max }, \forall i \in \mathcal{V} .
$$

(b) The BESSs must be able to supply/store the net charge demanded from them. Therefore, for all $T \geq 0$,

$S_{0}-\int_{0}^{T} \hat{P}_{n e t}^{p u}(t) d t \leq N s^{\max }, S_{0}-\int_{0}^{T} \hat{P}_{n e t}^{p u}(t) d t \geq N s^{\text {min }}$,

(c) The microgrid must not approach a steady state with zero demand. Therefore,

$$
\int_{0}^{T}\left|P_{n e t}^{p u}(t)\right| d t \rightarrow \infty \quad \text { as } \quad T \rightarrow \infty .
$$

If condition (c) is not satisfied, SoC synchronisation may require circulating currents.

The graph between the BESSs $\mathcal{G}(\mathcal{V}, \mathcal{E})$ is connected and bidirectional, as specified in Section II. It is assumed that $a_{\bar{i}}$ is chosen such that the distributed average current consensus protocol is much faster than the SoC dynamics. Therefore, the local estimates of the average per-unit current of the BESSs participating in the droop control can be treated as being equal to a common value, i.e. $\bar{i}_{L i}^{p u}(t)=\bar{i}_{L}^{p u *}(t), \forall i \in \mathcal{V}$. This is justified by the observation that even with low bandwidth communications, the speed of the dynamic average consensus protocol will be on the order of seconds, while at the highest practical rate a BESS will take minutes to be charged/discharged.

The following sliding mode controller is introduced for each BESS:

$$
u_{i}(t)= \begin{cases}U_{i}\left(\bar{i}_{L i}^{p u}(t), x_{i}(t)\right), & s_{i}(t) \geq \mathcal{A}_{i}(t) \text { and } \bar{i}_{L i}^{p u}(t)>0, \\ U_{i}\left(\bar{i}_{L i}^{p u}(t), x_{i}(t)\right), & s_{i}(t) \leq \mathcal{A}_{i}(t) \text { and } \bar{i}_{L i}^{p u}(t)<0, \\ 0, & \text { otherwise. }\end{cases}
$$

The closed-loop system belongs to the class of hybrid dynamical systems [33], [34]. Note that this controller is equivalent to $(6)$.

Finally, it is assumed that there exists a constant $\epsilon>0$, such that if $P_{n e t}^{p u}(t) \neq 0, \bar{i}_{L}^{p u *}(t) \in\left[-i_{L}^{\text {pumax }},-\epsilon\right] \cup\left[\epsilon, i_{L}^{\text {pumax }}\right]$. Otherwise, all of the BESSs would need to operate at their maximum charging or discharging current, and they would have no excess current capacity to use for SoC balancing (without introducing circulating currents).

Theorem A.1: Consider the system (7), (8) with the controller (16) and some functions $P_{n e t}^{p u}(t), \bar{i}_{L}^{p u *}(t)$. Suppose that the specified assumptions hold. Then, the constraint (12) holds, and $\lim _{t \rightarrow \infty}\left(s_{i}(t)-s_{j}(t)\right)=0$, for all $i, j$.

Proof of Theorem A.1: The following Lyapunov function is introduced (see [35]):

$$
V\left(s_{1}(t), s_{2}(t), \ldots, s_{N}(t)\right)=\sum_{i=1}^{N}\left|s_{i}(t)-\mathcal{A}_{i}(t)\right| .
$$

Clearly, $V\left(s_{1}(t), s_{2}(t), \ldots, s_{N}(t)\right) \geq 0$, and

$$
\begin{aligned}
& V\left(s_{1}(t), s_{2}(t), \ldots, s_{N}(t)\right)=0 \text { if and only if } \\
& s_{1}(t)=s_{2}(t)=\cdots=s_{N}(t) .
\end{aligned}
$$

Furthermore, let $\dot{V}\left(s_{1}(t), s_{2}(t), \ldots, s_{N}(t)\right)$ be the derivative of $V\left(s_{1}(t), s_{2}(t), \ldots, s_{N}(t)\right)$ along trajectories of the system (7), (8) with the controller (16). Then, it follows from the structure of the controller (16) that $\dot{V}\left(s_{1}(t), s_{2}(t), \ldots, s_{N}(t)\right)$ is always non-positive. Also, when $P_{n e t}^{p u}(t) \neq 0$ it follows that $\dot{V}\left(s_{1}(t), s_{2}(t), \ldots, s_{N}(t)\right) \leq-\frac{\epsilon}{N}$. This, with (15), implies that $V\left(s_{1}(t), s_{2}(t), \ldots, s_{N}(t)\right) \rightarrow 0$ as $t \rightarrow \infty$. Therefore,

$$
\lim _{t \rightarrow \infty}\left(s_{i}(t)-s_{j}(t)\right)=0 \forall i, j .
$$

The constraint (12) follows from the conditions in (13), (14). This completes the proof of Theorem A.1, i.e. the SoC levels of the distributed BESSs synchronise under the proposed control strategy.

\section{REFERENCES}

[1] C. Restrepo, A. Salazar, H. Schweizer, and A. Ginart, "Residential Battery Storage: Is the Timing Right?" IEEE Electrification Magazine, vol. 3, no. 3, pp. 14-21, Sep. 2015.

[2] M. S. Whittingham, "History, Evolution, and Future Status of Energy Storage," Proceedings of the IEEE, vol. 100, no. Special Centennial Issue, pp. 1518-1534, 2012.

[3] J. J. Justo, F. Mwasilu, J. Lee, and J.-W. Jung, "AC-microgrids versus DC-microgrids with distributed energy resources: A review," Renewable and Sustainable Energy Reviews, vol. 24, pp. 387-405, Aug. 2013.

[4] R. H. Lasseter, "MicroGrids," in 2002 IEEE Power Engineering Society Winter Meeting. Conference Proceedings (Cat. No.02CH37309), vol. 1, 2002, pp. 305-308.

[5] K. Strunz, E. Abbasi, and D. N. Huu, "DC Microgrid for Wind and Solar Power Integration," IEEE Journal of Emerging and Selected Topics in Power Electronics, vol. 2, no. 1, pp. 115-126, Mar. 2014.

[6] H. Ikebe, "Power Systems for Telecommunications in the IT Age," in Telecommunications Energy Conference, 2003. INTELEC '03. The 25th International, Oct. 2003, pp. 1-8.

[7] J. M. Guerrero, J. C. Vásquez, J. Matas, L. G. de Vicuna, and M. Castilla, "Hierarchical Control of Droop-Controlled AC and DC Microgrids-A General Approach Toward Standardization," IEEE Trans. Ind. Electron., vol. 58, no. 1, pp. 158-172, Jan. 2011.

[8] J. Schiffer, D. U. Sauer, H. Bindner, T. Cronin, P. Lundsager, and R. Kaiser, "Model prediction for ranking lead-acid batteries according to expected lifetime in renewable energy systems and autonomous powersupply systems," Journal of Power Sources, vol. 168, no. 1, pp. 66-78, May 2007.

[9] T. Morstyn, B. Hredzak, and V. G. Agelidis, "Distributed Cooperative Control of Microgrid Storage," IEEE Trans. Power Syst., vol. 30, no. 5, pp. 2780-2789, Oct. 2014.

[10] L. Meng, T. Dragičević, J. Vásquez, J. Guerrero, and E. R. Sanseverino, "Hierarchical control with virtual resistance optimization for efficiency enhancement and State-of-Charge balancing in DC microgrids," 2015 IEEE 1st International Conference on Direct Current Microgrids, ICDCM 2015, pp. 1-6, Jun. 2015. 
[11] T. Dragičević, J. M. Guerrero, J. C. Vásquez, and D. Skrlec, "Supervisory Control of an Adaptive-Droop Regulated DC Microgrid With Battery Management Capability," IEEE Trans. Power Electron., vol. 29, no. 2, pp. 695-706, Feb. 2014.

[12] S. D. J. McArthur, E. M. Davidson, V. M. Catterson, A. L. Dimeas, N. D. Hatziargyriou, F. Ponci, and T. Funabashi, "Multi-Agent Systems for Power Engineering Applications-Part I: Concepts, Approaches, and Technical Challenges," IEEE Trans. Power Syst., vol. 22, no. 4, pp. 1743-1752, Nov. 2007.

[13] Y. Simmhan, A. G. Kumbhare, B. Cao, and V. Prasanna, "An Analysis of Security and Privacy Issues in Smart Grid Software Architectures on Clouds," in 2011 IEEE 4th International Conference on Cloud Computing, Jul. 2011, pp. 582-589.

[14] F. L. Lewis, H. Zhang, K. Hengster-Movric, and A. Das, Cooperative Control of Multi-Agent Systems, ser. Communications and Control Engineering. London: Springer London, 2014.

[15] A. Bidram, A. Davoudi, F. L. Lewis, and J. M. Guerrero, "Distributed Cooperative Secondary Control of Microgrids Using Feedback Linearization," IEEE Trans. Power Syst., vol. 28, no. 3, pp. 3462-3470, Aug. 2013.

[16] J. W. Simpson-Porco, F. Dörfler, and F. Bullo, "Synchronization and power sharing for droop-controlled inverters in islanded microgrids," Automatica, vol. 49, no. 9, pp. 2603-2611, Sep. 2013

[17] V. Nasirian, S. Moayedi, A. Davoudi, and F. L. Lewis, "Distributed Cooperative Control of DC Microgrids," IEEE Trans. Power Electron., vol. 30 , no. 4, pp. 2288-2303, Apr. 2015.

[18] J. Zhao and F. Dörfler, "Distributed control and optimization in DC microgrids," Automatica, vol. 61, pp. 18-26, Aug. 2015.

[19] D. Florian, J. W. Simpson-porco, and F. Bullo, "Breaking the hierarchy: Distributed control \& economic optimality in microgrids," IEEE Trans. Control Netw. Syst., vol. PP, no. 99, pp. 1-1, 2014.

[20] S. Bolognani and S. Zampieri, "A Distributed Control Strategy for Reactive Power Compensation in Smart Microgrids," IEEE Trans. Autom. Control, vol. 58, no. 11, pp. 2818-2833, Nov. 2013.

[21] T. Dragičević, J. M. Guerrero, and J. C. Vásquez, "A Distributed Control Strategy for Coordination of an Autonomous LVDC Microgrid Based on Power-Line Signaling," IEEE Trans. Ind. Electron., vol. 61, no. 7, pp. 3313-3326, Jul. 2014

[22] T. Dragičević, X. Lu, J. C. Vásquez, and J. M. Guerrero, "DC Microgrids-Part I: A Review of Control Strategies and Stabilization Techniques," IEEE Trans. Power Electron., vol. 31, no. 7, pp. 4876 - 4891, Sep. 2015.

[23] T. Morstyn, B. Hredzak, G. D. Demetriades, and V. G. Agelidis, "Unified Distributed Control for DC Microgrid Operating Modes," IEEE Trans. Power Syst., vol. 31, no. 1, pp. 802-812, Mar. 2015.

[24] T. Morstyn, B. Hredzak, and V. G. Agelidis, "Cooperative MultiAgent Control of Heterogeneous Storage Devices Distributed in a DC Microgrid," IEEE Trans. Power Syst., vol. 31, no. 4, pp. 2974-2986, Sep. 2015.

[25] T. Morstyn, B. Hredzak, V. G. Agelidis, and G. Demetriades, "Cooperative control of DC microgrid storage for energy balancing and equal power sharing," in 2014 Australasian Universities Power Engineering Conference (AUPEC), Sep. 2014, pp. 1-6.

[26] T. Morstyn, B. Hredzak, and V. G. Agelidis, "Communication delay robustness for multi-agent state of charge balancing between distributed AC microgrid storage systems," in 2015 IEEE Conference on Control Applications (CCA), Sep. 2015, pp. 181-186.

[27] C. Li, D. Dragicevic, M. G. Plaza, F. Andrade, J. C. Vásquez, and J. M. Guerrero, "Multiagent based distributed control for state-of-charge balance of distributed energy storage in DC microgrids," in IECON 2014 - 40th Annual Conference of the IEEE Industrial Electronics Society, Oct. 2014, pp. 2180-2184.

[28] C. Li, T. Dragičević, J. C. Vásquez, J. M. Guerrero, and E. A. A. Coelho, "Multi-agent-based distributed state of charge balancing control for distributed energy storage units in AC microgrids," in 2015 IEEE Applied Power Electronics Conference and Exposition (APEC), Mar. 2015, pp. 2967-2973.

[29] P. Sanchis, A. Ursaea, E. Gubia, and L. Marroyo, "Boost DC-AC Inverter: A New Control Strategy," IEEE Trans. Power Electron., vol. 20, no. 2, pp. 343-353, Mar. 2005.

[30] R. Olfati-saber, D. P. Spanos, and R. M. Murray, "Dynamic Consensus for Mobile Networks," in 2005 IFAC World Congress, Jul. 2005.

[31] J.-J. E. Slotine and W. Li, Applied Nonlinear Control. Englewood Cliffs, New Jersey: Prentice Hall, 1991.

[32] T. Kim and W. Qiao, "A Hybrid Battery Model Capable of Capturing Dynamic Circuit Characteristics and Nonlinear Capacity Effects," IEEE Trans. Energy Convers., vol. 26, no. 4, pp. 1172-1180, Dec. 2011.
[33] A. S. Matveev and A. V. Savkin, Qualitative Theory of Hybrid Dynamical Systems. Boston: Birkhauser, 2000.

[34] A. V. Savkin and R. J. Evans, Hybrid Dynamical Systems: Controller and Sensor Switching Problems. Boston: Birkhauser, 2002.

[35] I. R. Petersen, V. A. Ugrinovskii, and A. V. Savkin, Robust Control Design Using H-infinity Methods. London: Springer-Verlag, 2000.

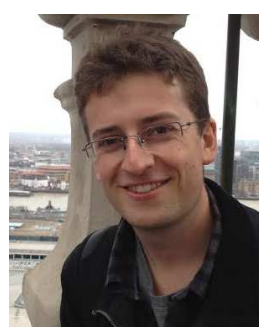

Thomas Morstyn (S'14-M'16) received the B.E. (Hon.) degree from the University of Melbourne, Australia, in 2011, and the PhD degree from the University of New South Wales, Australia, in 2016, both in electrical engineering.

He worked as an electrical engineer in the Rio Tinto Technology and Innovation Group between 2012 and 2014. He is currently an Oxford Martin Fellow in the Department of Engineering Science at the University of Oxford. His current research interests include multi-agent control and optimisation for the integration of distributed renewable generation and energy storage systems into power networks.

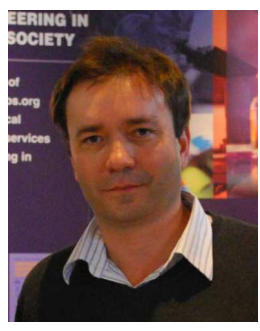

Andrey V. Savkin (M'97-SM'98) received the M.S. degree in mathematics and the Ph.D. degree in applied mathematics from The Leningrad University, Leningrad, Russia, in 1987 and 1991, respectively. Since 2000, he has been a Professor in the School of Electrical Engineering and Telecommunications, The University of New South Wales, Sydney, NSW, Australia. His current research interests include robust control and filtering, robotics, networked control systems, control of power systems and the application of control and signal processing to biomedical engineering and medicine. Dr. Savkin has been an associate editor for several international journals and conferences.

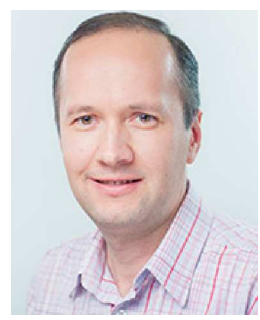

Branislav Hredzak (M'98-SM'13) received the B.Sc./M.Sc. degree from the Technical University of Kosice, Slovak Republic, in 1993, and the Ph.D. degree from Napier University of Edinburgh, U.K., in 1997, all in electrical engineering.

He was a Lecturer and a Senior Researcher in Singapore from 1997 to 2007 . He is currently a Senior Lecturer in the School of Electrical Engineering and Telecommunications, The University of New South Wales, Sydney, NSW, Australia. His current research interests include hybrid storage technologies and advanced control systems for power electronics and storage systems.

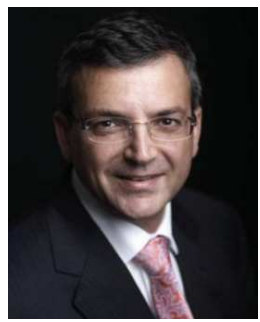

Vassilios G. Agelidis (S'89-M'91-SM'00-F'16) was born in Serres, Greece. He received the B.Eng. degree in electrical engineering from the Democritus University of Thrace, Thrace, Greece, in 1988, the M.S. degree in applied science from Concordia University, Montreal, QC, Canada, in 1992, and the $\mathrm{Ph} . \mathrm{D}$. degree in electrical engineering from Curtin University, Perth, Australia, in 1997. He has worked at Curtin University (1993-1999), University of Glasgow, U.K. (2000-2004), Murdoch University, Perth, Australia (2005-2006), the University of Sydney, Australia (2007-2010), and the University of New South Wales (UNSW), Sydney, Australia (2010-2016). He is currently a professor at the Department of Electrical Engineering, Technical University of Denmark.

Dr. Agelidis received the Advanced Research Fellowship from the U.K.'s Engineering and Physical Sciences Research Council in 2004. He was the Vice-President Operations within the IEEE Power Electronics Society from 2006 to 2007. He was an AdCom Member of the IEEE Power Electronics Society from 2007 to 2009 and the Technical Chair of the 39th IEEE Power Electronics Specialists Conference, Rhodes, Greece, 2008. 